\title{
Four-dimensional polymer collapse II: Interacting self-avoiding trails
}

\author{
T. Prellberg $\dagger^{*}$ and A. L. Owczarek $\ddagger$ \\ $\dagger$ Institut für Theoretische Physik, \\ Technische Universität Clausthal, \\ Arnold Sommerfeld Straße 6, \\ D-38678 Clausthal-Zellerfeld, \\ Germany \\ $\ddagger$ Department of Mathematics and Statistics, \\ The University of Melbourne, \\ 3010, Victoria, \\ Australia.
}

April 9, 2001

\begin{abstract}
We have simulated four-dimensional interacting self-avoiding trails (ISAT) on the hypercubic lattice with standard interactions at a wide range of temperatures up to length 4096 and at some temperatures up to length 16384. The results confirm the earlier prediction (using data from a non-standard model at a single temperature) of a collapse phase transition occurring at finite temperature. Moreover they are in accord with the phenomenological theory originally proposed by Lifshitz, Grosberg and Khokhlov in three dimensions and recently given new impetus by its use in the description of simulational results for fourdimensional interacting self-avoiding walks (ISAW). In fact, we argue that the available data is consistent with the conclusion that the collapse transitions of ISAT and ISAW lie in the same universality class, in contradiction with long-standing predictions. We deduce that there exists a pseudo-first order transition for ISAT in four dimensions at finite lengths while the thermodynamic limit is described by the standard polymer mean-field theory (giving a second-order transition), in contradiction to the prediction that the upper critical dimension for ISAT is $d_{u}=4$.
\end{abstract}

PACS numbers: 05.50.+q, 05.70.fh, 61.41.+e

Key words: Interacting self-avoiding trails, polymer collapse, coil-globule transition, four dimensions.

*email: thomas.prellberg@tu-clausthal.de, Phone: +49-5323 72 2085, Fax: +49-5323 723799 


\section{Introduction}

The geometric collapse of polymers in dilute solution, being one of the fundamental and most well studied phase transitions in statistical mechanics attracts continuing interest from both the theoretician attempting to understand the subtleties inherent in this phenomenon and the experimentalist wanting a firm foundation for interpreting their results on more complicated systems. One approach has been the use of lattice models of various types of self-avoiding paths to describe the geometry of linear polymers with added local potentials to broadly account for the complex solvent-polymer and polymer-polymer interactions. The canonical lattice path utilised in this regard has been the self-avoiding walk (SAW) as it explicitly demonstrates the "excluded volume" expected of physical polymers. However, much of our understanding of three-dimensional polymer collapse [1, 2, 3] has come from calculations using the continuum Edwards model 44, 5] which is based upon intersecting random paths, rather than interacting self-avoiding walks (ISAW). Hence it is of interest to study lattice models closer in nature to the Edwards model. One such model is based on the lattice paths known as self-avoiding trails (SAT) [6, 7, 8, 9] rather than self-avoiding walks. The type of interaction considered (contact versus nearest-neighbour for ISAW) is also more closely analogous to the Edwards model. It is also worth noting that the upper critical dimension for polymer collapse is expected to be $d_{u}=3$, and therefore many subtle logarithmic corrections are predicted to occur in three dimensions. As is often the case in the study of critical phenomena our understanding is enhanced by considering the dimension of the problem as a parameter that can be varied. Any discrepancies between the behaviour of competing models in four dimensions for example would impinge on our interpretation of results in three dimensions.

Self-avoiding trails (SAT) or trails for short are paths on a lattice which have no two steps on the same bond of that lattice but may occupy the same site. This restriction is sometimes referred to as bond-avoiding, in contrast to self-avoiding walks (SAW) which are site-avoiding (that is, no two vertices of the walk may occupy the same site on the lattice). Clearly walks are, by default, also bond-avoiding. Trails possess an excluded volume effect and it is fairly well established that SAT and SAW are in the same universality class [10, 11] which describes good solvent polymers. (For a more recent discussion of the subtle differences between walks and trails regarding corrections to scaling see [12].) It has been shown [0] that there should exist a collapse transition when contact attraction is added to the trail model: this model of polymer collapse is known as interacting self-avoiding trails (ISAT). Moreover, Shapir and Oono [7] have argued that this point should be tricritical in nature, as it is at the ISAW collapse point. However, they predict that ISAW and ISAT are in different universality classes. Importantly, while the upper critical dimension for ISAW is expected to be $d_{u}=3$, the Shapir-Oono field theory gives $d_{u}=4$ for ISAT. Therefore, this implies generically that logarithmic corrections occur at the $\theta$-point in four dimensions, which presumably should occur at a finite temperature. On the other hand the above discussion about the hypothesised equivalence of the critical phenomena of self-avoiding 
walks and the Edwards model may lead one to the opposite conclusion, namely that the collapse transitions of ISAT, ISAW and the (3-parameter) Edwards model lie in the same universality class. Computer simulations in two and three dimensions have given conflicting results and so it is of interest to ascertain whether computer simulations in four dimensions may shed light on the question of whether ISAW and ISAT share the same collapse universality class.

Recent developments have been two-fold. Firstly, preliminary evidence of collapse in any polymer model in four dimensions was presented in [13] using the so-called kinetic growth trail which effectively simulates ISAT with a particular fixed set of non-standard Boltzmann weights (and so fixed temperature). It was argued from the results of the simulations that this set of Boltzmann weights corresponded to a so-called $\theta$-point where the ISAT behaves in a way predicted, by the mean-field theory of the polymer collapse, to be precisely at the collapse transition point. Plausibility arguments then deduced the existence of the collapse transition as the temperature is varied through this value. Secondly, and more startling have been the results of simulations [14, 15] of interacting self-avoiding walks in four dimensions. A dramatic collapse transition was located in this model using simulations up to length 32678 . The nature of this transition was described as a pseudo-first order transition because at any finite length the distribution of the internal energy was bimodal when the temperature was near that at which the specific heat attained its maximum (one indicator of the transition's position), while it was argued that the effective latent heat would slowly decay to zero in the thermodynamic limit (as the length diverges) with an anomalous exponent. On the other hand the specific heat seemed to diverge as the length was increased. Despite these peculiar findings the simulational results were able to be interpreted in terms of a framework explained some time ago by Khokhlov [16], who applied the non-standard mean-field approach of Lifshitz, Grosberg and Khokhlov (LGK) [17, 18, 19] to arbitrary dimensions. This theory is based on a phenomenological free energy in which the competition between a bulk free energy of a dense globule and its surface tension drive the transition. The consequences of this surface free energy were largely ignored in the polymer literature until recently, when its effect on the scaling form of the finite-size partition function was proposed and confirmed [20, 21, 22, 23]. While markedly different for finite lengths this theory still predicts a standard Gaussian $\theta$-point and in the infinite length limit a second order phase transition with a jump in the specific heat (no divergence as the temperature is varied: the divergence as length is varied exists but "disappears" in the thermodynamic limit).

In this work we have simulated the standard ISAT model on the four-dimensional hypercubic lattice over a range of temperatures using the PERM algorithm [24] in a similar fashion to simulations implemented for ISAW [14]. This algorithm is particularly efficient in high dimensions and so is well suited to these simulations. We find evidence that parallels the findings in the ISAW simulations 14 for a collapse transition with the signature of pseudo-first order type. The transition is, if anything more pronounced, with an equivalent strength at shorter lengths. We demonstrate that our data is at least as, if not more, consistent with the LGK theory as is the ISAW results [14]. Hence we deduce that the collapse transitions of ISAW and ISAT in 
four dimensions are in the same universality class. It follows from these conclusions that the upper critical dimension for collapse in ISAT is $d_{u}<4$, and that possibly $d_{u}=3$ in accord with the standard polymer theory and with the logarithmically modified Gaussian state found in three-dimensional kinetic growth trail simulations [25].

\section{The ISAT model and scaling theories}

\subsection{The ISAT model}

We define the ISAT model on the four-dimensional hypercubic lattice in the following way. The lattice has coordination number 8 and we consider configurations $\varphi_{N}$ of trails, or bond-avoiding walks, of length $N$ (bonds) starting from a fixed origin. Let $m_{k}, k=1, \ldots, 4$ be the number of sites of the lattice that has been visited $k$ times by the trail so that $\sum k m_{k}=N+1$. The partition function of a very general interacting trail model is

$$
Z_{N}\left(\omega_{2}, \omega_{3}, \omega_{4}\right)=\sum_{\varphi_{N}} \omega_{2}^{m_{2}} \omega_{3}^{m_{3}} \omega_{4}^{m_{4}}
$$

where $\omega_{k}$ is the Boltzmann weight associated with $k$-visited sites. The canonical model is one where every segment of the trail at some contact site interacts with every other segment at that site, so that

$$
\omega_{k}=\omega^{\left(\begin{array}{c}
k \\
2
\end{array}\right)} \text { for } k=2,3,4
$$

with $\omega \equiv \omega_{2}$. This implies that in our specific case

$$
\omega_{2}=\omega, \quad \omega_{3}=\omega^{3}, \quad \omega_{4}=\omega^{6} .
$$

The Boltzmann weight $\omega=e^{\beta \epsilon}$ is associated with a energy of contact $-\epsilon$ so that $\omega>1.0$ for attractive interactions. As we are only interested in the attractive case here one can set $\epsilon=1$ for convenience. We define a reduced finite-size free energy per step $\kappa_{N}(\omega)$ as

$$
\kappa_{N}(\omega)=\frac{1}{N} \log Z_{N}(\omega)
$$

The usual free energy is related to this by $-\beta F_{N} \equiv N \kappa_{N}(\omega)$.

The average of any quantity $Q$ over the ensemble set of allowed paths $\Omega_{N}$ of length $N$ is given generically by

$$
\langle Q\rangle_{N}(\omega)=\frac{\sum_{\varphi \in \Omega_{N}} Q(\varphi) \omega^{m(\varphi)}}{\sum_{\varphi \in \Omega_{N}} \omega^{m(\varphi)}}
$$

where $m=m_{2}+3 m_{3}+6 m_{4}$. We define a normalised finite-size internal energy per step by

$$
U_{N}(\omega)=\frac{\langle m\rangle}{N}
$$


and a normalised finite-size specific heat per step by

$$
C_{N}(\omega)=\frac{\left\langle m^{2}\right\rangle-\langle m\rangle^{2}}{N}
$$

These quantities are related in the usual way to the reduced free energy via $U_{N}=\partial \kappa_{N} / \partial \log \omega$ and $C_{N}=\partial U_{N} / \partial \log \omega$. Note that because of the absent factors of temperature both $U_{N}$ and $C_{N}$ can take on non-zero values for $\omega=1.0$.

The thermodynamic limit in this problem is given by the limit $N \rightarrow \infty$ so that the thermodynamic free energy per step $f_{\infty}(\omega)$ is given by

$$
-\beta f_{\infty}(\omega)=\kappa_{\infty}(\omega)=\lim _{N \rightarrow \infty} \kappa_{N}(\omega)
$$

This quantity determines the partition function asymptotics, i.e. $Z_{N}(\omega)$ grows to leading order exponentially as $\mu(\omega)^{N}$ with $\mu(\omega)=e^{\kappa_{\infty}(\omega)}$.

In our simulations we calculated two measures of the polymer's average size. Firstly, specifying a trail by the sequence of position vectors $\mathbf{r}_{0}, \mathbf{r}_{1}, \ldots, \mathbf{r}_{N}$ the average mean-square end-to-end distance is

$$
\left\langle R_{e}^{2}\right\rangle_{N}=\left\langle\left(\mathbf{r}_{N}-\mathbf{r}_{0}\right) \cdot\left(\mathbf{r}_{N}-\mathbf{r}_{0}\right)\right\rangle
$$

We shall use the symbol $R_{e, N}^{2}$ to be equivalent to

$$
R_{e, N}^{2}(\omega) \equiv\left\langle R_{e}^{2}\right\rangle_{N}
$$

The mean-square distance of a site occupied by the trail to the endpoint, $\mathbf{r}_{0}$, is given by

$$
\left\langle R_{m}^{2}\right\rangle_{N}=\frac{1}{N+1} \sum_{i=0}^{N}\left\langle\left(\mathbf{r}_{i}-\mathbf{r}_{0}\right) \cdot\left(\mathbf{r}_{i}-\mathbf{r}_{0}\right)\right\rangle .
$$

Again we define

$$
R_{m, N}^{2}(\omega) \equiv\left\langle R_{m}^{2}\right\rangle_{N}
$$

We also define the ratio

$$
B_{N}(\omega)=\frac{R_{m, N}^{2}}{R_{e, N}^{2}}
$$

which should have a universal limit in each critical phase of the model.

\subsection{Standard polymer scaling theory}

Let us expand our view in this section to general polymer scaling theory. We first assume that there is a single collapse transition at some value of temperature and explore the four-dimensional behaviour we might expect from the above defined quantities in each of the phases. The basic physics of the coil-globule (collapse) transition can be understood by the consideration of the 
average size of the polymer, $R_{N}$, either $R_{e, N}$ or $R_{m, N}$, as a function of length $N$ in each of the phases, so let us consider this first. Let us define an effective Boltzmann weight $\omega=e^{\beta}$-of course, in any particular model or physical system the associated potentials may be different. Generally one always expects that

$$
R_{N}^{2} \sim a(\omega) N^{2 \nu} \quad \text { as } \quad N \rightarrow \infty
$$

for any fixed value of $\omega$. In four dimensions at infinite temperature, $\omega=1$, it has been predicted [26] that

$$
R_{N}^{2} \sim a^{+} N(\log (N))^{1 / 4}
$$

If there does exist a collapse transition then one would expect that this scaling extends (with a constant $a^{+}$that depends on temperature) down to the transition point. In the collapsed phase the polymer is expected to assume a dense configuration on average and hence the globular value of the radius-of-gyration exponent is $\nu_{g}=1 / d=1 / 4$ [1] with

$$
R_{N}^{2} \sim a^{-}(\omega) N^{1 / 2}
$$

Finally at some finite transition temperature $1.0<\omega_{t}<\infty$ a Gaussian scaling of the radius of gyration should occur, that is

$$
R_{N}^{2} \sim a^{\theta} N
$$

so that $\nu_{t}=1 / 2$. This Gaussian scaling is often used (theoretically at least) to define the $\theta$-point $\omega=\omega_{\theta}$ of an isolated polymer so that $\omega_{t}=\omega_{\theta}$. The universal ratio $B_{N}$ is expected to converge to the value $B_{\infty}=1 / 2$ both in the swollen phase and at $\omega_{\theta}$. However, one would expect slow logarithmic corrections for $\omega<\omega_{\theta}$ and algebraic corrections at $\omega_{\theta}$. For $\omega>\omega_{\theta}$ the phase is no longer expected to be critical and so $B_{\infty}$ is no longer universal and may be a non-constant function of $\omega$.

One can also consider the scaling of the partition function in each of the regimes, given that there is a transition. For high temperatures $1.0<\omega<\omega_{\theta}$ one expects the infinite temperature behaviour, which is [26]

$$
Z_{N} \sim b^{+}(\omega) \mu(\omega)^{N}(\log N)^{1 / 4},
$$

while at low temperatures [20] one expects asymptotics of the form

$$
Z_{N} \sim b^{-}(\omega) \mu(\omega)^{N} \mu_{s}(\omega)^{N^{3 / 4}} N^{g}
$$

where $\mu_{s}$ is related to the surface free energy of the polymer globule and the exponent $g$ need not be universal (we only write it for completeness of the asymptotic form). For $\omega=\omega_{\theta}$ one expects

$$
Z_{N} \sim b^{\theta} \mu\left(\omega_{\theta}\right)^{N}
$$


as a reflection of Gaussian behaviour.

In the thermodynamic limit the thermodynamic functions $f_{\infty}(\omega), U_{\infty}(\omega)$ and $C_{\infty}(\omega)$ are all expected to be analytic functions of $\omega$ except at $\omega_{\theta}$. By using the correspondence to the tricritical model [1] the mean field theory would imply that the specific heat had a jump discontinuity at $\omega_{\theta}$ (the associated exponent $\alpha=0$ ). Of course, for finite $N$ there is no sharp transition for an isolated polymer (unless one examines a macroscopic number of such polymers).

\subsection{LGK theory}

We now provide a brief review of the predictions of the theory of Lifshitz, Grosberg and Khokhlov (LGK) [19 as applied to four-dimensional polymer collapse by Khokhlov 16]. Firstly, there exists a state where the excluded volume property of long chain molecules is exactly cancelled by the attractive interactions between parts of the polymer as mediated by the solvent. This is the $\theta$-state. Secondly, when the attraction becomes even stronger there eventuates a globular state where the polymer behaves as a liquid drop. The results of the theory are based on a phenomenological free energy of that globular state relative to the free energy of the pure Gaussian state of the $\theta$-point at $T_{\theta}$. Hence the condition applied to find the finite-size position of the transition is to equate the relative free energy to zero. The relative free energy is given as a sum of bulk and surface contributions which are, in turn, given in terms of the second and third virial coefficients, the length of the chains, and the linear size of the polymer found from the globular density. In particular both the bulk and surface free energies are proportional to the square of the second virial coefficient. It is assumed that on approaching the $\theta$-point the second virial coefficient goes to zero linearly with temperature while the third virial coefficient remains non-zero. Note that this implies a quadratic dependence of the bulk free energy on the distance to the $\theta$-point. Since the free energy has exponent $2-\alpha$ this implies an exponent $\alpha=0$ (assuming that this part of the free energy is singular). Therefore a second-order phase transition occurs in the thermodynamic limit.

It is further assumed that the density in the globule is proportional to the second virial coefficient and hence also goes to zero linearly with temperature on approaching the $\theta$-point $(\beta=1)$. Again using an effective Boltzmann weight $\omega=e^{\beta}$ and defining the transition as when the free energy is zero, Khokhlov [16] finds a finite-size transition temperature $\omega_{c, N}$ which approaches the $\theta$-temperature $\left(\omega_{\theta}\right)$ as

$$
\omega_{c, N}-\omega_{\theta} \sim \frac{s}{N^{1 / 3}}
$$

for some constant $s$. That is, the polymer collapse shift exponent is $1 / 3$. The width of the transition region $\Delta \omega$ at finite $N$ can be found from the free energy rewritten in terms of this

\footnotetext{
${ }^{1}$ for the sake of ease of expression in this section we will use the word "temperature" to mean the effective Boltzmann weight
} 
transition temperature to scale as

$$
\Delta \omega \sim \frac{w}{N^{2 / 3}}
$$

for some constant $w$. That is, the polymer collapse crossover exponent is $2 / 3$. Hence note that the size of the crossover region is asymptotically small relative to the shift of the transition.

Following the work [15] of Lifshitz, Grosberg and Khokhlov [19] one can also calculate the change in the internal energy over the crossover width of the transition $\Delta \omega$ as the latent heat (or "heat of the transition") by using expression of the free energy in terms of the transition temperature as

$$
\Delta U \sim \frac{u^{c}}{N^{1 / 3}}
$$

The corresponding height of the peak in the specific heat is

$$
C_{N}\left(\omega_{c, N}\right) \sim h^{c} N^{1 / 3}
$$

So to summarise the LGK picture, the theory predicts a thermodynamic second-order transition at a Gaussian $\theta$-point with a jump in the specific heat. For finite polymer length this transition is shifted below the $\theta$-point by a temperature of the order of $O\left(N^{-1 / 3}\right)$ with the width of the transition of the order of $O\left(N^{-2 / 3}\right)$. Over this width there is a rapid change in the internal energy that scales as $O\left(N^{-1 / 3}\right)$ : the important point here of course is that this tends to zero for infinite length so the effect of the peak in the specific heat is scaled away for $N$ large, leaving a finite jump in the thermodynamic limit. To understand this further let us consider the distribution of internal energy as a function of temperature and length. For any $\omega$ below $\omega_{\theta}$ and well above $\omega_{c, N}$ one expects the distribution of internal energy to look like a single peaked distribution centred close to the thermodynamic limit value: a Gaussian distribution is expected around the peak with variance $O\left(N^{-1 / 2}\right)$. In fact, this picture should be valid for all temperatures outside the range $\left[\omega_{c, N}-O\left(N^{-2 / 3}\right), \omega_{c, N}+O\left(N^{-2 / 3}\right)\right]$. When we enter this region we expect to see a double peaked distribution as in a first-order transition region. For any temperature in this region there should be two peaks in the internal energy distribution separated by a gap $\delta U$ of the order of $\delta U \approx \Delta U \propto O\left(N^{-1 / 3}\right)$. Each peak is of Gaussian type with individual variances again of the order of $O\left(N^{-1 / 2}\right)$. Hence as $N$ increases the peaks will become more and more distinct and relatively sharper but the peak positions will be getting closer together. We refer to this scenario as a pseudo-first-order transition or, more correctly, as first-order-like finite-size corrections to a second-order phase transition. If there were a real first-order transition then the distance between the peaks should converge to a non-zero constant. On the other hand the transition is not a conventional second-order phase transition with a well defined limit distribution of the internal energy that is simply bimodal. 


\section{Simulational results and analysis}

We have simulated ISAT on a 4-dimensional hyper-cubic lattice using the Pruned-Enriched Rosenbluth Method (PERM), a clever generalisation of a simple kinetic growth algorithm [24, 27. PERM builds upon the Rosenbluth-Rosenbluth method [28], in which trails are generated by simply growing an existing trail kinetically but overcomes the exponential "attrition" and re-weighting needed in this approach by a combination of enrichment and pruning strategies. Our implementation here follows our previous ISAW work [15, 14]. Briefly, we chose upper and lower thresholds $W^{u}$ and $W^{l}$, for enrichment and pruning respectively, proportional to the current estimate of the average weight of a trail at length $N,\left\langle Z_{N}\right\rangle / s_{N}$, where $s_{N}$ is the number of generated samples at length $N$, and $\left\langle Z_{N}\right\rangle$ is the current estimate of the partition function at length $N$. That is to say, $W_{N}^{u}=c_{N}^{u}\left\langle Z_{N}\right\rangle / s_{N}, W_{N}^{l}=c_{N}^{l}\left\langle Z_{N}\right\rangle / s_{N}$. In order to enforce an even sample size distribution we allowed for dynamic adjustment of $c_{N}^{u}$ and $c_{N}^{l}$, keeping the quotient of the thresholds $Q=c_{N}^{u} / c_{N}^{l}$ constant. To stabilise the dynamic adjustment, we enforced $c_{N}^{u}>c_{\min }^{u}$ and $c_{N}^{l}<c_{\max }^{l}$. As in the ISAW work, we chose $c_{\min }^{u}=2$ and $c_{\max }^{l}=1 / 2$. For each run, we attempted to choose the smallest threshold quotient $Q$ for which we could obtain an even sample size distribution.

Each run had a maximum length $N_{\max }$ set and while individual runs gave information about shorter lengths we collected data from independent runs at some shorter lengths to guarantee statistical independence. Simulations were conducted with the maximum lengths $N_{\max }$ set to $512,1024,2048$, and 4096 , with values of $\omega$ ranging from 1.0 to 2.07 for $N_{\max }=512$, from 1.0 to 2.00 for $N_{\max }=1024$, from 1.0 to 1.78 for $N_{\max }=2048$, from 1.0 to 1.67 for $N_{\max }=4096$. We also ran many closer spaced simulations in the range of $\omega$ from 1.4 to 1.42 at length $N_{\max }=16384$. At each fixed $\omega$, we generated at least $10^{7}$ trails. To illustrate the computational effort, the generation of a sample of size $10^{7}$ at length $N_{\max }=16384$ took about 2 weeks CPU time on a $600 \mathrm{MHz}$ DEC Alpha. The threshold quotient $Q$ used ranged from 10 to 80 with larger values of $Q$ needed for higher $\omega$. We also performed one large-scale simulation deep in the collapsed regime, with $N_{\max }=512$ and $\omega=4.0$, for which it was necessary to increase the threshold quotient up to $Q=1000$.

We computed statistics for $R_{e, N}^{2}$ and $R_{m, N}^{2}$, the partition function $Z_{N}$, the internal energy $U_{N}$ and specific heat $C_{N}$. Moreover, we generated the distribution of the number of interactions at $N_{\max }$. The distributions obtained at various temperatures were then combined using the multiple histogram method [29].

The disadvantage of PERM is that due to the enrichment the generated data is not independent. All the data generated during one "tour", i.e. between two successive returns of the algorithm to length 0 , is correlated. Therefore, we kept track of the statistics of tour sizes $t$ to get a rough idea of the quality of the data. In our statistical evaluation we use (somewhat arbitrarily) the quotient of $s_{N}$ and $\sqrt{\left\langle t^{2}\right\rangle}$ as a measure of an effective independent sample size. This is correct as long as the tour sizes don't fluctuate too strongly, and, more importantly, 
as long as individual tours explore the sample space evenly. When simulating in the collapsed phase, both of these assumptions break down, and the sample is dominated by few huge tours. Moreover, the pruning and enrichment rates become so large that the efficiency of the algorithm is significantly decreased. Error bars are only given for high temperature and $\theta$-point simulation figures (figures 1 and 3) and are based on the method described above, although we always computed error estimates. No error bars are given in the rest of the figures because of the performance of the algorithm in and near the collapsed phase, even though the data seemed converged sufficiently.

Let us first discuss the scaling of the mean-squared end-to-end distance normalised by trail length, $R_{e, N}^{2} / N$ and the mean-squared distance of a site occupied by the trail to the trail's endpoint, $R_{m, N}^{2} / N$. In the swollen phase, our results are in correspondence with the logarithmic corrections seen by Grassberger [30]. As in that paper, we observe that $R_{e, N}^{2}$ grows faster than $N$ for $\omega$ near 1.0, and fitting to $N(\log N)^{c}$ at $\omega=1.0$ gives an effective exponent close to that predicted by field theory $(1 / 4)$. This value shifts as $\omega$ is increased indicating the presence of strong temperature-dependent correction terms.

By considering when the quantity $R_{e, N}^{2} / N$ approaches a constant we narrowed our search for the $\theta$-point to the region $\omega=1.40$ to $\omega=1.42$. In this region we extended our simulations to trails of length 16384. Figure 1 shows a plot of $R_{e, N}^{2} / N$ versus $1 / N$ for values of $\omega$ between 1.408 and 1.420. At $\omega=1.414(3)$ we have an approximate linear asymptotic dependence of $R_{e, N}^{2}$ on $N$. Moreover, at $\omega=1.414$ we estimate from our data $B_{N}=R_{m, N}^{2} / R_{e, N}^{2}=0.5000(2)$, which is also indicative of Gaussian behaviour: the precision of this estimate stems from the weakness of the corrections to scaling at this point.

As shown in Figure 2 for $\omega=4.0, R_{e, N}^{2}$ changes non-monotonically in $N$ ! After an initial increase, the size of the polymer actually starts to shrink around $N=50$ as it undergoes collapse corresponding to a rapid increase of the density. For large enough $N$, we expect to see the true collapsed behaviour, i.e. $R_{e, N}^{2}$ growing again as $N^{1 / 2}$, but while we see $R_{e, N}^{2}$ just starting to increase again, the asymptotic regime is beyond the reach of our PERM simulations on current computer hardware.

Let us now discuss the scaling of the partition function. The swollen phase and the $\theta$-point behaviour can also be clearly identified from the free-energy scaling. In the swollen phase we find again the same behaviour as [30]. The presence of logarithmic corrections is consistent with our data. At $\omega=1$, we estimate $\mu(1)=\mu_{S A T}=6.926080(2)$. In the $\theta$-region, an analysis shows that here $Z_{N}$ scales as $\mu^{N}$ with weak $1 / N$ corrections. Figure 3 shows $Z_{N} / Z_{N / 2}^{2}$ plotted versus $1 / N$ from which we estimate the $\theta$-point to be $\omega_{\theta}=1.414(3)$ and $\mu_{\theta}=7.0016(6)$. (At fixed $\omega$, the accuracy is of course higher: for $\omega=1.414$, we estimate $\mu=7.0015714(5)$.) In the collapsed region, one expects the finite-size free energy to have a strong correction term of the order $N^{-1 / 4}$ due to surface effects. Figure 4 shows this for $\omega=4.0$. As argued above, the globule starts to collapse when the length is above $N=50$, and we notice here the onset of a corresponding strong change in the behaviour of the finite-size free energy around this length $\left(N^{-1 / 4} \approx 0.35\right)$. Even 
though we cannot simulate long enough chain lengths to clearly determine the precise nature of the correction term, our data is certainly compatible with a $N^{-1 / 4}$ correction for $N^{-1 / 4}<0.3$ (i.e. $N>150$ ).

In order to study the collapse transition more closely, we now focus our attention on the internal energy and specific heat. As can be seen from Figure 5, the specific heat has a sharply peaked graph for each length that becomes more sharply peaked as $N$ increases. The transition region becomes sharper and stays well separated from the $\theta$-point, even though the location of the transition (peak in the specific heat) approaches the $\theta$-point slowly. The scaling of the shift of the transition towards the $\theta$-point, $\omega_{c, N}-\omega_{\theta}$, and the sharpening of the transition width, $\Delta \omega$, are both shown in Figure 6. Here, we defined the location of the collapse transition by the location of the specific heat peak, and the width of the transition is given by the interval in which the specific heat is greater or equal to half the value of the peak height. Expecting from the LGK theory that $\omega_{c, N}-\omega_{\theta}$ scales as $N^{-1 / 3}$ and that $\Delta \omega$ scales as $N^{-2 / 3}$, we plot both $N^{1 / 3}\left(\omega_{c, N}-\omega_{\theta}\right)$ and $\Delta \omega N^{2 / 3}$ versus $N^{-2 / 3}$ which was chosen empirically. Both quantities can be seen to be asymptotic to constants: on the graph extrapolations give non-zero intercepts. Hence, Figure 6 shows that the LGK predictions are compatible with our simulations. We do note that the corrections to scaling for $\Delta \omega$ are much larger than for $\omega_{c, N}-\omega_{\theta}$.

The character of the transition becomes apparent if one plots the internal energy density distribution (rescaled density of interactions) at the finite-size collapse transition, $\omega_{c, N}$. Figure 07 shows the emergence of a bimodal distribution. At length 512 one sees a slight non-convexity, which at length 4096 has evolved into a distribution dominated by two sharp and well-separated peaks. The values of the minima and maxima of the distribution are different by two orders of magnitude. This bimodal distribution means that as $\omega$ is increased through the transition region the density distribution switches from the peak located at a small value of contacts to the peak located at a larger value of contacts, corresponding to a sudden change in the internal energy. In the collapsed phase, the width of the peak is much wider than in the swollen phase, implying a larger specific heat. It is this difference between the swollen and collapsed phases' specific heats that will eventually become the thermodynamic second order jump. The rapid first-order like switch between two peaks in the distribution becomes more pronounced at larger polymer lengths since the depth of the "valley" between the two peaks becomes relatively larger.

Continuing with the scaling predictions from Khoklov theory, a suitably defined finite-size latent heat, $\Delta Q$, should tend to zero as $N^{-1 / 3}$ in the thermodynamic limit. One possible measure of this latent heat is given by the product of specific heat peak $C_{N}\left(\omega_{c, N}\right)$ and specific heat width $\Delta \omega$, and another is given by the distance $\delta U$ of the peaks in the bimodal internal energy distribution. Figure 8 shows the behaviour of both of these quantities. One notices two things from this figure. Firstly, it indicates that $C_{N}\left(\omega_{c, N}\right) \Delta \omega$ decreases to zero linearly in $N^{-1 / 3}$ as predicted. However, even at length $N=2048\left(N^{-1 / 3} \approx 0.08\right)$ there is considerable discrepancy between the two quantities plotted, so that one needs to be cautious in the interpretation of the scaling behaviour. The explanation for the discrepancy between the two quantities is of course 
that in order to observe the asymptotic behaviour the two peaks in the histogram have to be well separated and distinct, and that this is only really the case when $N$ is of the order of $10^{3}$. We caution that Figure 8 alone is not sufficient to discriminate between the scenario proposed here and a real first-order transition in the thermodynamic limit, but we believe the rest of our data and other theoretical facts provide a more consistent picture.

When comparing our data with the simulations for ISAW [15] we note further that the bimodal distribution emerges for ISAT at much shorter configurations, so that the peaks in the distribution for ISAT at length $N=512$ are already more pronounced than the peaks in the distribution for ISAW at length $N=2048$. To quantify this observation, we turn to the scaling predictions of LGK theory. An important parameter in the theory is the quotient $a^{d} / v$, where $a$ is the mean-square distance between two subsequent monomers (repeated unit element of the polymer: equivalent to occupied sites of the lattice model) along a chain and $v$ is the effective excluded volume of a monomer, defined via the vanishing of the second virial coefficient at the $\theta$-temperature. For instance, the shift of the transition temperature (cf. equation 2.21) is given more explicitly by

$$
\frac{\omega_{c, N}-\omega_{\theta}}{\omega_{\theta}} \sim\left(\frac{\tilde{s} a^{4}}{N v}\right)^{1 / 3}
$$

where $\tilde{s}$ is a constant proportional to the quotient of the third virial coeffficient and the excluded volume squared. From Figure we estimate that $N^{1 / 3}\left(\omega_{c, N}-\omega_{\theta}\right)$ asymptotes to 3.4(1) for ISAT, and for ISAW we estimate for the same quantity the value 0.92(3) [15]. Identifying $a$ with the lattice constant, which in both models is set equal to one, we can get a rough estimate for the relative size of the effective excluded volume $v$ in both models. We obtain

$$
\frac{v_{S A T}}{v_{S A W}} \approx 0.03 \frac{\tilde{s}_{S A T}}{\tilde{s}_{S A W}}
$$

and thereby quantify the intuitive notion that the excluded volume effect is numerically "weaker" in trails than in walks, though of the same basic type.

In conclusion, our ISAT simulations elucidate further the structure of the polymer collapse transition in four dimensions. We show conclusively that there is indeed a collapse transition at a finite temperature. Secondly, we find evidence for a $\theta$-temperature at which the polymer is well approximated by Gaussian behaviour as well as for a collapse transition which is well separated from the $\theta$-point. The collapse transition shows many first-order like features, such as a bimodal distribution in the internal energy. An analysis of the scaling behaviour of this transition in the context of the theory of Lifshitz, Grosberg and Khokhlov 19, 16] shows that a consistent interpretation of these findings is that of first-order like finite-size corrections to a thermodynamic second-order transition. These finding are essentially the same as those made recently for ISAW collapse in four dimensions [14]. Consequently, we deduce that the upper critical dimension for ISAT is $d_{u}<4$ (most likely 3 ) and not $d_{u}=4$ as was previously predicted. 


\section{Acknowledgements}

Financial support from the Australian Research Council is gratefully acknowledged by ALO.

\section{References}

[1] P.-G. de Gennes, J. Physique Lett. 36, L55 (1975).

[2] M. J. Stephen, Phys. Lett. A. 53, 363 (1975).

[3] B. Duplantier, J. Physique 43, 991 (1982).

[4] B. Duplantier, Europhys. Lett. 1, 491 (1986).

[5] B. Duplantier, J. Chem. Phys. 86, 4233 (1987).

[6] A. Malakis, Physica 84, 256 (1976).

[7] Y. Shapir and Y. Oono, J. Phys. A. 17, L39 (1984).

[8] H. A. Lim, A. Guha, and Y. Shapir, J. Phys. A 21, 773 (1988).

[9] I. S. Chang, A. Guha, H. A. Lim, and Y. Shapir, J. Phys. A 21, L559 (1988).

[10] A. J. Guttmann, J. Phys. A. 18, 567 (1985).

[11] A. J. Guttmann, J. Phys. A. 18, 575 (1985).

[12] I. Guim, H. W. J. Blöte, and T. W. Burkhardt, J. Phys. A. 30, 413 (1997).

[13] A. L. Owczarek and T. Prellberg., Physica A 260, 20 (1998).

[14] A. L. Owczarek and T. Prellberg., Europhys. Lett. 51, 602 (2000).

[15] T. Prellberg and A. L. Owczarek, Phys. Rev. E. 62, 3780 (2000).

[16] A. R. Khokhlov, Physica A. 105, 357 (1981).

[17] I. M. Lifshitz, Zh. Eksp. Teor. Fiz. 55, 2408 (1968), [Sov. Phys. - JETP, 28, 545, 1969].

[18] I. M. Lifshitz, A. Y. Grosberg, and A. R. Khokhlov, Zh. Eksp. Teor. Fiz. 71, 1634 (1976), [Sov. Phys. - JETP, 44, 1976].

[19] I. M. Lifshitz, A. Y. Grosberg, and A. R. Khokhlov, Rev. Mod. Phys. 50, 683 (1978).

[20] A. L. Owczarek, T. Prellberg, and R. Brak, Phys. Rev. Lett. 70, 951 (1993).

[21] A. L. Owczarek, J. Phys. A. 26, L647 (1993).

[22] P. Grassberger and R. Hegger, J. Chem. Phys. 102, 6881 (1995). 
[23] P. P. Nidras, J. Phys. A. 29, 7929 (1996).

[24] P. Grassberger, Phys. Rev. E 56, 3682 (1997).

[25] T. Prellberg and A. L. Owczarek, Phys. Rev. E. 51, 2142 (1995).

[26] P.-G. de Gennes, Scaling Concepts in Polymer Physics, Cornell University Press, Ithaca, 1979.

[27] H. Frauenkron, U. Bastolla, E. Gerstner, P. Grassberger, and W. Nadler, Phys. Rev. Lett. 80, 3149 (1998).

[28] M. N. Rosenbluth and A. W. Rosenbluth, J. Chem. Phys. 23, 356 (1955).

[29] A. M. Ferrenberg, Phys. Rev. Lett. 61, 2635 (1988).

[30] P. Grassberger, R. Hegger, and L. Schäfer, J. Phys. A. 27, 7265 (1994). 
Figure 1: $R_{e, N}^{2} / N$ versus $1 / N$ in the $\theta$-region: $\omega=1.408,1.411,1.414,1.417,1.420$ from top to bottom.

Figure 2: $R_{e, N}^{2}$ versus $N$ for $\omega=4.0$ up to length 512 .

Figure 3: $Z_{N} / Z_{N / 2}^{2}$ versus $1 / N$ in the $\theta$-region: $\omega=1.408,1.411,1.414,1.417,1.420$ from bottom to top.

Figure 4: Finite-size free energy $\kappa_{N}$ versus $N^{-1 / 4}$ for $\omega=4.0$.

Figure 5: Specific heat $C_{N}$ versus $\omega$ for lengths 512, 1024, 2048, and 4096 from right to left respectively, using the multi-histogram method.

Figure 6: Scaling of the transition: shift and width of the collapse region. Shown are the scaling combinations $N^{1 / 3}\left(\omega_{c, N}-\omega_{\theta}\right)$ and $N^{2 / 3} \Delta \omega$ versus $N^{-2 / 3}$.

Figure 7: Internal energy density distributions at $\omega_{c, N}$ for 512 and 4096. The more highly peaked distribution is associated with length 4096.

Figure 8: Scaling of the latent heat $\Delta U$ : our two measures of $\Delta U, C_{N}\left(\omega_{c, N}\right) \Delta \omega$ and peak distance $\delta U$ are plotted versus $N^{-1 / 3}$. 


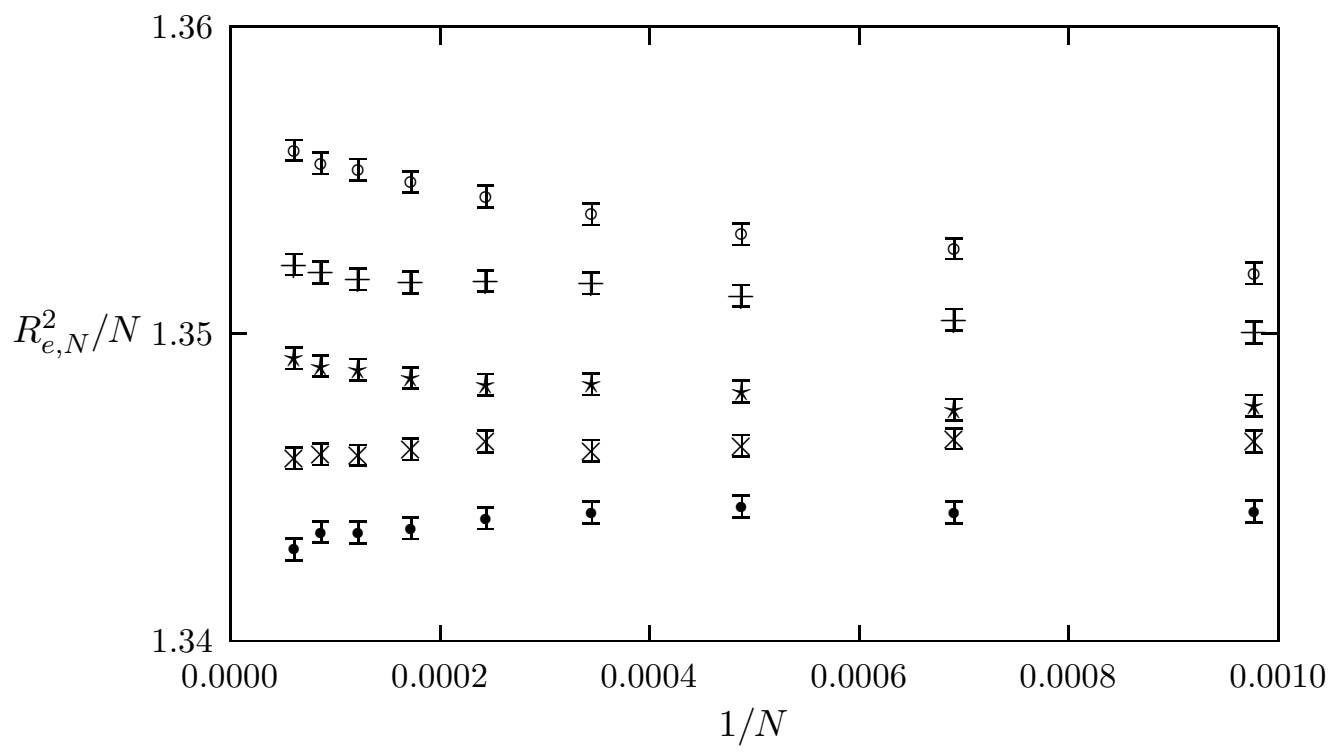

Figure 1: $R_{e, N}^{2} / N$ versus $1 / N$ in the $\theta$-region: $\omega=1.408,1.411,1.414,1.417,1.420$ from top to bottom. 


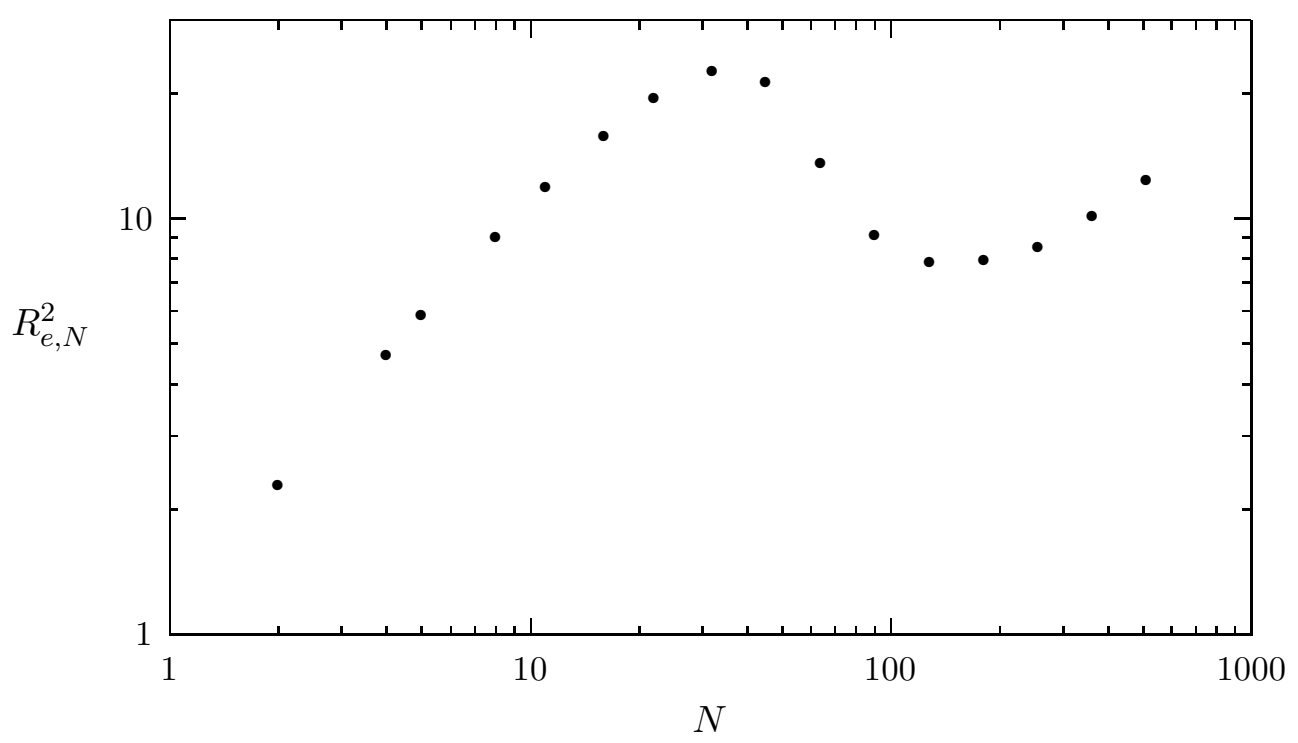

Figure 2: $R_{e, N}^{2}$ versus $N$ for $\omega=4.0$ up to length 512 . 


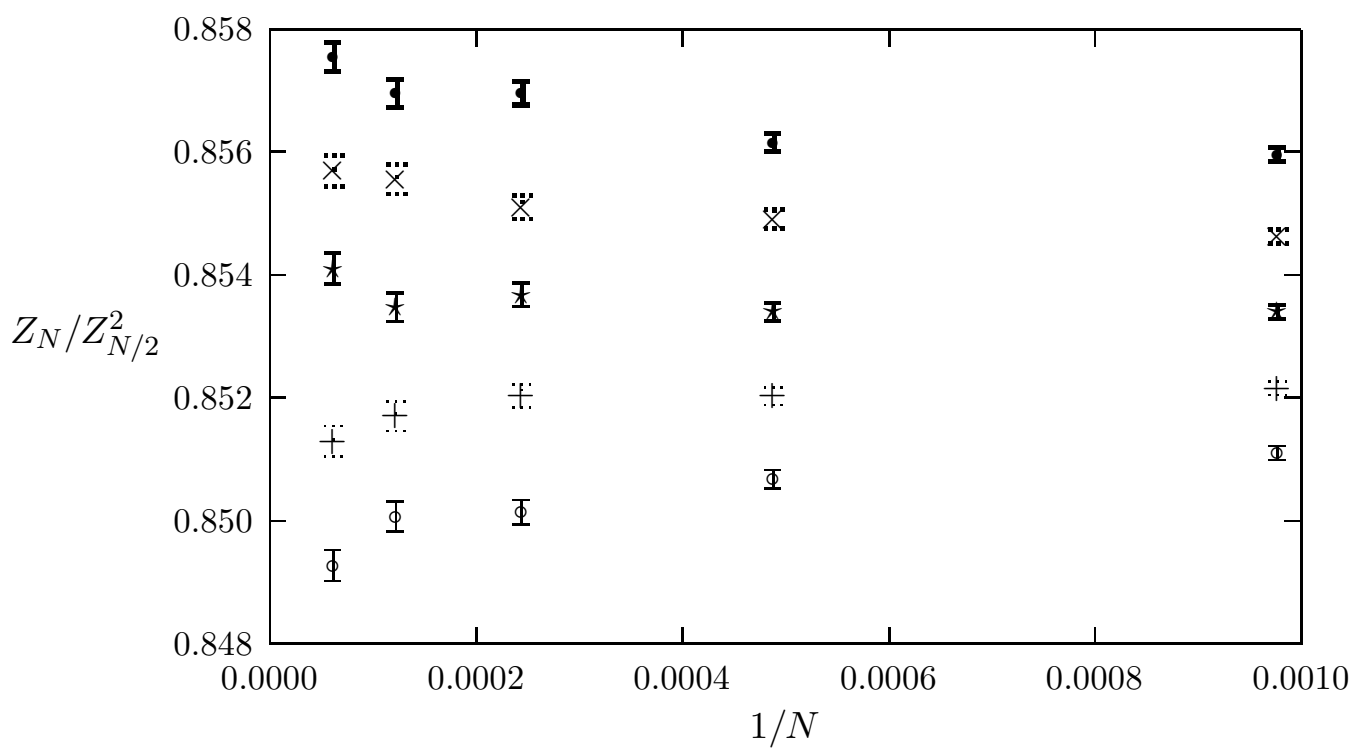

Figure $3: Z_{N} / Z_{N / 2}^{2}$ versus $1 / N$ in the $\theta$-region: $\omega=1.408,1.411,1.414,1.417,1.420$ from bottom to top. 


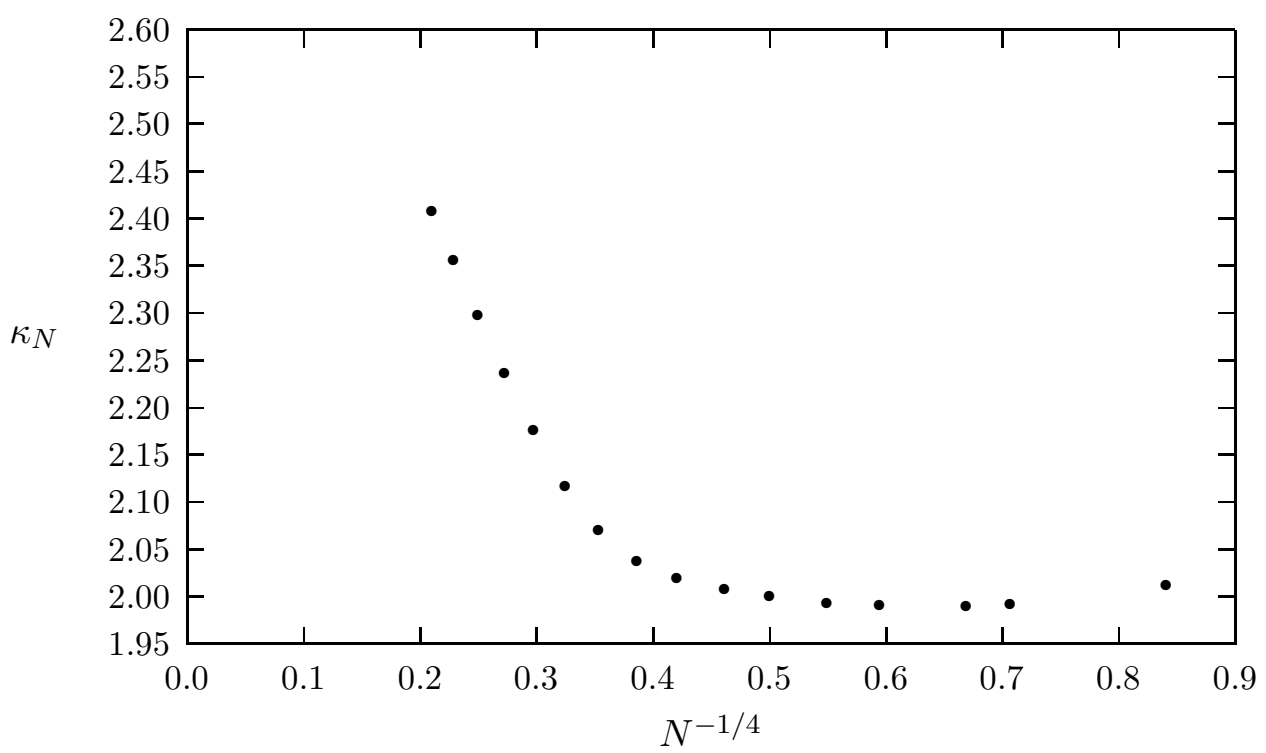

Figure 4: Finite-size free energy $\kappa_{N}$ versus $N^{-1 / 4}$ for $\omega=4.0$. 


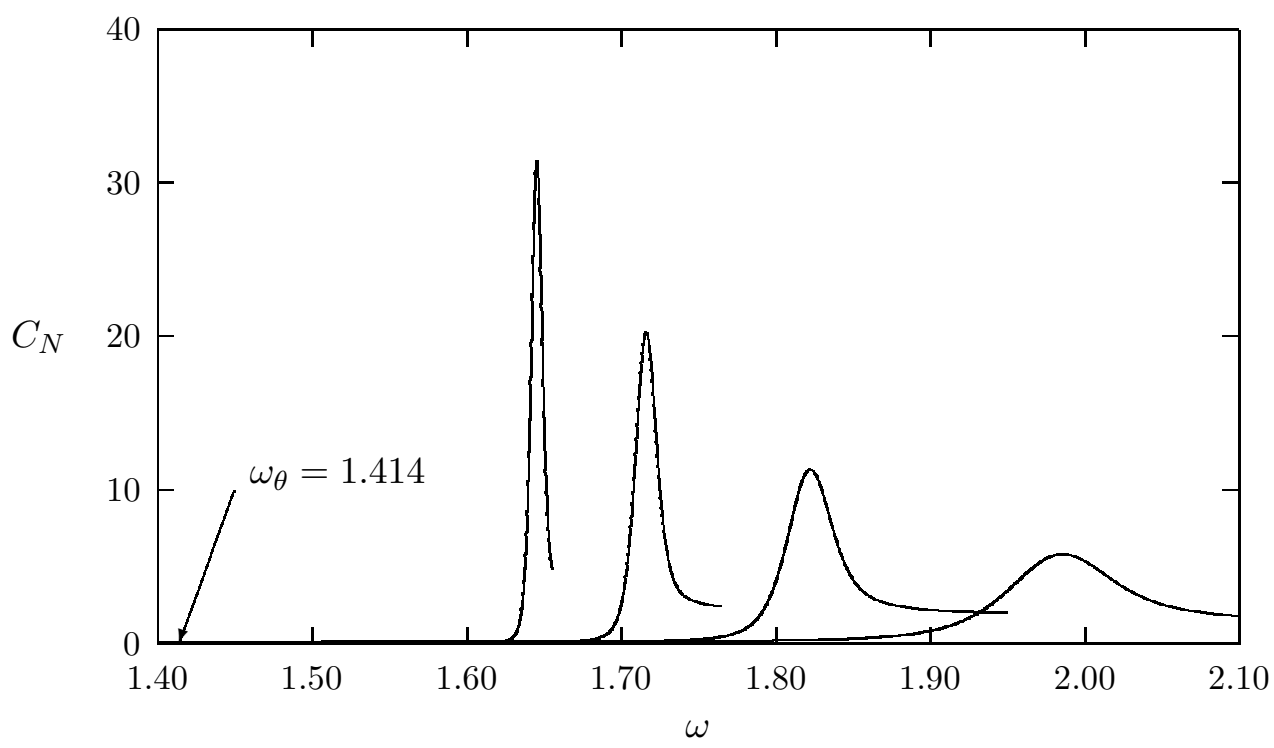

Figure 5: Specific heat $C_{N}$ versus $\omega$ for lengths 512, 1024, 2048, and 4096 from right to left respectively, using the multi-histogram method. 


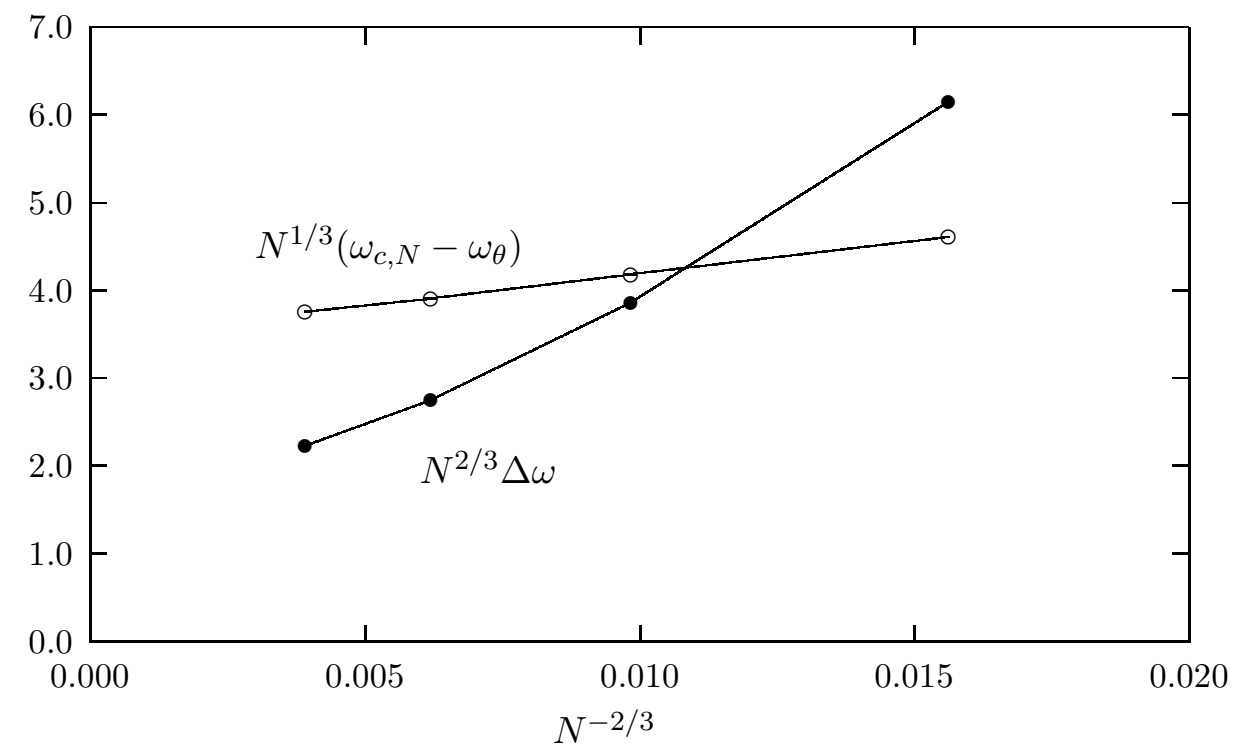

Figure 6: Scaling of the transition: shift and width of the collapse region. Shown are the scaling combinations $N^{1 / 3}\left(\omega_{c, N}-\omega_{\theta}\right)$ and $N^{2 / 3} \Delta \omega$ versus $N^{-2 / 3}$. 


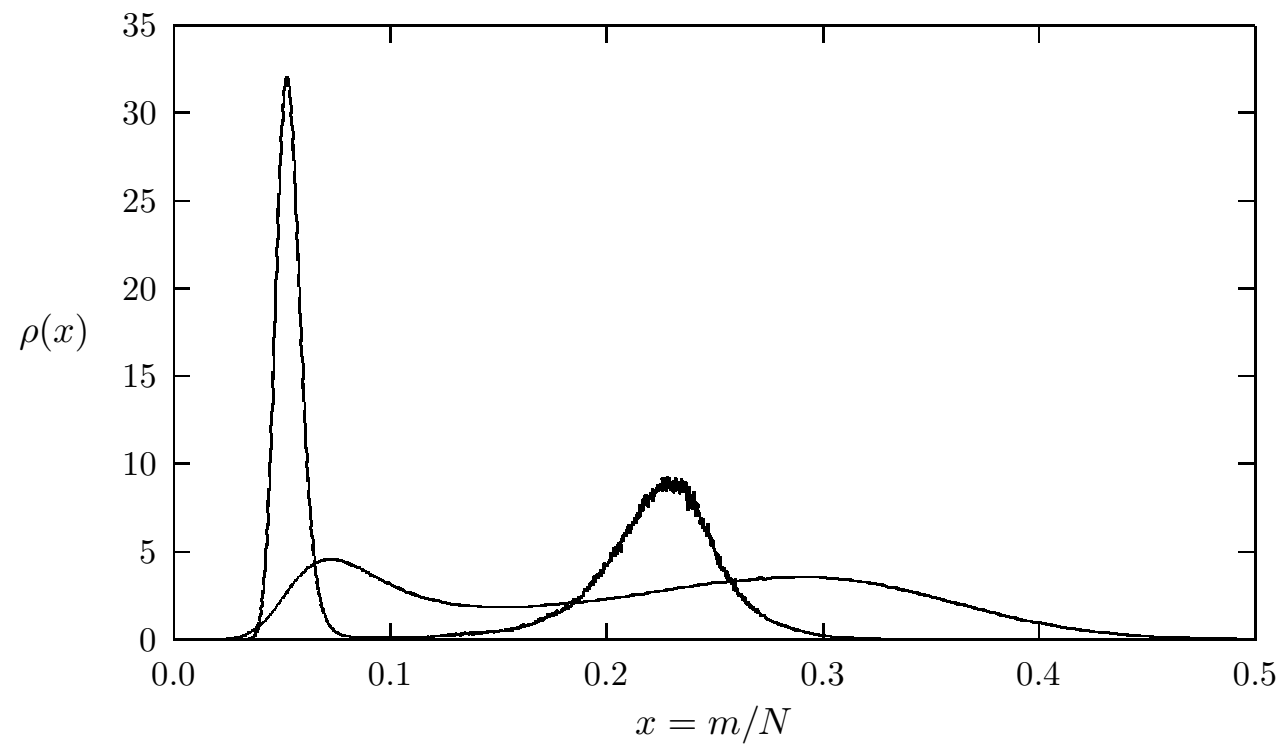

Figure 7: Internal energy density distributions at $\omega_{c, N}$ for 512 and 4096. The more highly peaked distribution is associated with length 4096. 


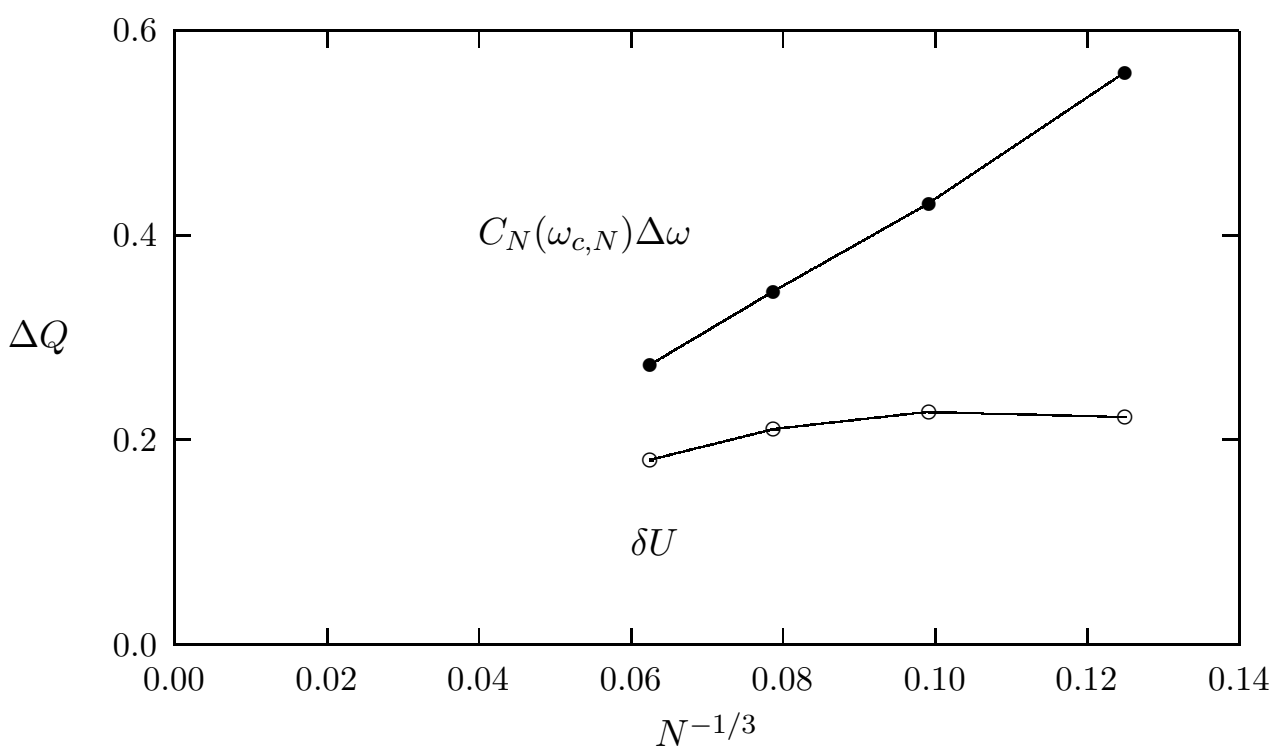

Figure 8: Scaling of the latent heat $\Delta U$ : our two measures of $\Delta U, C_{N}\left(\omega_{c, N}\right) \Delta \omega$ and peak distance $\delta U$ are plotted versus $N^{-1 / 3}$. 